\title{
HIGHER COMMUTATIVITY AND NILPOTENCY IN FINITE GROUPS.
}

\author{
E. TORRES-GIESE
}

\begin{abstract}
We consider ordered tuples in finite groups generating nilpotent subgroups. Given an integer $q$ we consider the poset of nilpotent subgroups of class less than $q$ and its corresponding coset poset. These posets give rise to a family of finite Dirichlet series parametrized by the nilpotency class of the subgroups, which in turn reflect probabilistic and topological invariants determined by these subgroups. Connections of these series to filtrations of classifying spaces of a group are discussed.
\end{abstract}

\section{INTRODUCTION}

In 1936 P. Hall addressed to some extent the problem of computing the probability that a randomly chosen ordered $s$-tuple of elements in a finite group $G$ generates the group. Hall proved that this probability, denoted by $P(G, s)$, can be expressed as a finite Dirichlet series. For instance (see [5]),

$$
P(P S L(2,7), s)=1-\frac{14}{7^{s}}-\frac{8}{8^{s}}+\frac{21}{21^{s}}+\frac{28}{28^{s}}+\frac{56}{56^{s}}-\frac{84}{84^{s}} .
$$

Hall's results allow us to think of this as a function over the complex numbers. In 4 ] this function was related to the coset poset of a finite group. This poset is the set of all proper cosets of a group ordered by inclusion. If we denote this poset by $\mathcal{C}(G)$, then Brown (following Bouc) proved that

$$
P(G,-1)=1-\chi(\mathcal{C}(G)) .
$$

The reciprocal of $P(G, s)$ is called the probabilistic zeta function of $G$ (see [5]).

We can twist the probability question to ask the following: given a finite group $G$, what is the probability that a randomly chosen ordered $s$-tuple generates an abelian subgroup of $G$ ? If we denote this probability by $P_{2}(G, s)$, then we have

$$
P_{2}(G, s)=\frac{\left|\operatorname{Hom}\left(\mathbb{Z}^{s}, G\right)\right|}{|G|^{s}}
$$

since the set of ordered commuting $s$-tuples in $G$ can be identified with the set of group homomorphisms $\operatorname{Hom}\left(\mathbb{Z}^{s}, G\right)$. The number $P_{2}(G, s)$ was also studied in [9] under the name of multiple commutativity degree. The set (space if $G$ has some topology) $\operatorname{Hom}\left(\mathbb{Z}^{s}, G\right)$ appears in different contexts of mathematics such as Differential Geometry, Group Cohomology, and $K$-theory (see [1]). The sets $\operatorname{Hom}\left(\mathbb{Z}^{s}, G\right), s \geq 0$, can be assembled to form a simplicial space. The realization of this simplicial space is denoted by $B(2, G)$ and it is the first layer of a filtration of the classifying space $B G$. The space $B(2, G)$ turns out to capture delicate information about the group $G$. For instance, when $G$ is finite of odd order the pull-back

Date: November 19, 2018.

Key words and phrases. Dirichlet series, coset poset, commuting elements, probabilistic zeta function, conjugacy classes, commutativity degree. 
fibration $E(2, G) \rightarrow B(2, G)$, obtained from the universal $G$-bundle over $B G$, captures the celebrated Feit-Thompson Theorem; and from the point of view of group cohomology, it can be seen that there is an $F$-monomorphism $H^{*}\left(B G ; \mathbb{F}_{p}\right) \rightarrow H^{*}\left(B(2, G) ; \mathbb{F}_{p}\right)$. In this paper we will show that the space $B(2, G)$ is closely related to the probability function $P_{2}(G, s)$.

Let $\mathcal{N}_{2}(G)$ be the poset of all abelian subgroups of $G$. It was proved in $[2$ that the space $B(2, G)$ is a homotopy colimit, namely

$$
B(2, G) \simeq \operatorname{hocolim}_{A \in \mathcal{N}_{2}(G)} B A
$$

and this can be used to show that

$$
E(2, G) \simeq \operatorname{hocolim}_{A \in \mathcal{N}_{2}(G)} G / A .
$$

For instance, if $q=2^{n}$ and $n \geq 2$, then

$$
B\left(2, S L\left(2, \mathbb{F}_{q}\right)\right) \simeq\left[\bigvee^{q+1} B(\mathbb{Z} / q)^{n}\right] \vee\left[\bigvee^{\frac{1}{2} q(q-1)} B \mathbb{Z} /(q+1)\right] \vee\left[\bigvee^{\frac{1}{2} q(q+1)} B \mathbb{Z} /(q-1)\right]
$$

and

$$
E\left(2, S L\left(2, \mathbb{F}_{q}\right)\right)=\underset{\left(q^{2}-1\right)^{2}(q+1)-q^{2}\left(q^{2}+1\right)+1}{S^{1}}
$$

Lets define $\mathcal{C}_{2}(G)$ as the poset of all cosets of proper abelian subgroups. In this context we have the following result

Theorem 1.1. If $G$ is a finite group, then

(1) there is a homotopy equivalence $\mathcal{C}_{2}(G) \simeq E(2, G)$,

(2) $P_{2}(G, s)$ is a finite Dirichlet series, and

(3) $P_{2}(G,-1)=\chi(E(2, G))$.

For instance,

$$
P_{2}\left(A_{5}, s\right)=\frac{6}{12^{s}}+\frac{5}{15^{s}}+\frac{10}{20^{s}}-\frac{20}{60^{s}} .
$$

The poset $\mathcal{C}_{2}(G)$ has some intriguing properties. Brown in his paper 4] asked: Can we characterize finite solvable groups in terms of the combinatorial topology of the coset poset? The answer is yes for groups of odd order if instead we consider the poset $\mathcal{C}_{2}(G)$ since, as noted in [2], the following statements are equivalent

(1) Every group of odd order is solvable.

(2) The map $H_{1}\left(\mathcal{C}_{2}(G)\right) \rightarrow H_{1}(B(2, G))$ is not onto for every group $G$ of odd order.

This probabilistic setting can be extended to higher classes of nilpotency. We will show that there is a finite Dirichlet series whose value at a positive integer $s$ is equal to

$$
\frac{\mid\left\{\left(g_{1}, \ldots, g_{s}\right) \in G^{s}: \Gamma^{q}\left(\left\langle g_{1}, \ldots, g_{s}\right\rangle\right\}=1 \mid\right.}{|G|^{s}}
$$

where $\Gamma^{q}$ stands for the $q^{\text {th }}$-stage of the lower central series. Note that this latter ratio is precisely the probability that a randomly chosen $s$-tuple generates a nilpotent subgroup of class less than $q$. The paper is organized as follows: in Section 2 we cover some background; in Section 3 we relate the coset posets to probabilistic invariants via Möbius functions; in Section 4 we explore some properties of the finite 
Dirichlet series afforded by nilpotent subgroups; in Section 5 we provide an explicit formula to compute the number of commuting elements in a symmetric group; and in Section 6 we present upper and lower bounds for the probabilistic invariants aforementioned. In this paper a group will always be finite unless otherwise stated.

\section{Preliminaries}

Recall that the lower central series of a group $K$ is defined inductively by $\Gamma^{1}(K)=K$ and $\Gamma^{q}(K)=$ $\left[\Gamma^{q-1}(K), K\right]$. We say that a group $K$ is nilpotent of class $c$ if $\Gamma^{c+1}(K)=1$ and $\Gamma^{q}(K) \neq 1$ for $q \leq c$. By convention we will write $\Gamma^{\infty}(K)=1$. Let $G$ be a group, and fix $q \geq 2$. We define

$$
B_{n}(G, q)=\operatorname{Hom}\left(F_{n} / \Gamma_{n}^{q}, G\right)
$$

and

$$
E_{n}(G, q)=G \times \operatorname{Hom}\left(F_{n} / \Gamma_{n}^{q}, G\right),
$$

where $F_{n}$ is the free group on $n$ generators and $\Gamma_{n}^{q}=\Gamma^{q}\left(F_{n}\right)$. Note that $B_{n}(G, q) \subseteq G^{n}$ and $E_{n}(G, q) \subseteq$ $G^{n+1}$. The following functions provide the sets $B_{*}(G, q)$ and $E_{*}(G, q)$ with a simplicial structure: $d_{i}$ : $E_{n}(q, G) \rightarrow E_{n-1}(q, G)$ for $0 \leq i \leq n$, and $s_{j}: E_{n}(q, G) \rightarrow E_{n+1}(q, G)$ for $0 \leq j \leq n$, are given by

$$
d_{i}\left(g_{0}, \ldots, g_{n}\right)=\left\{\begin{array}{lr}
\left(g_{0}, \ldots, g_{i} \cdot g_{i+1}, \ldots, g_{n}\right) & 0 \leq i<n, \\
\left(g_{0}, \ldots, g_{n-1}\right) & i=n,
\end{array}\right.
$$

and

$$
s_{j}\left(g_{0}, \ldots, g_{n}\right)=\left(g_{0}, \ldots, g_{j}, e, g_{j+1}, \ldots, g_{n}\right)
$$

for $0 \leq j \leq n$. Similarly, we have maps $d_{i}$ and $s_{j}$ for $B_{*}(q, G)$ defined in the same way, except that the first coordinate $g_{0}$ is omitted and the map $d_{0}$ takes the form $d_{0}\left(g_{1}, \ldots, g_{n}\right)=\left(g_{2}, \ldots, g_{n}\right)$. Note that $G$ acts on $E_{*}(G, q)$ by multiplication on the first coordinate $g\left(g_{0}, g_{1} \ldots, g_{n}\right)=\left(g g_{0}, g_{1}, \ldots, g_{n}\right)$, with orbit space homeomorphic to $B_{*}(q, G)$. We denote by $B(q, G)$ and $E(q, G)$ the geometric realizations of the aforementioned simplicial sets. Thus we have $G$-bundles $E(q, G) \rightarrow B(q, G)$ for each $q \geq 2$, and fibrations $E(q, G) \rightarrow B(q, G) \rightarrow B G$. Unlike the classical situation, the space $E(q, G)$ is not necessarily contractible. Note that if $E(q, G)$ is contractible, then $B(q, G) \simeq B G$. We can also consider the simplicial set of $B N_{*}(G)$ whose set of $n$-simplices is defined as

$$
\left\{\left(g_{1}, \ldots, g_{n}\right) \mid\left\langle g_{1}, \ldots, g_{n}\right\rangle \neq G\right\} .
$$

Likewise, we can define $N E_{*}(G)$ as the simplicial set whose $n$-simplices is defined as $G \times B N_{n}(G)$. The simplicial structure of these latter two spaces is the same as that of $B(q, G)$ and $E(q, G)$ respectively. We will denote their realization by $B N(G)$ and $E N(G)$. Note that there is a fibration $E N(G) \rightarrow B N(G) \rightarrow$ $B G$.

The inclusions $F_{n} / \Gamma_{n}^{q+1} \subseteq F_{n} / \Gamma_{n}^{q}$ induce the following filtrations:

$$
E(2, G) \subseteq \cdots \subseteq E(q, G) \subseteq E(q+1, G) \subseteq \cdots \subseteq E(\infty, G)=E G,
$$

and

$$
B(2, G) \subseteq \cdots \subseteq B(q, G) \subseteq B(q+1, G) \subseteq \cdots \subseteq B(\infty, G)=B G .
$$

Moreover, $B(q, G) \subseteq B N(G) \subset B G$, and $E(q, G) \subseteq E N(G) \subset E G$ for all $q \geq 2$. 
Definition 2.1. Let $q \geq 2$. We define $\mathcal{N}_{q}(G)$ as the poset of all nilpotent, proper subgroups of $G$ of class $<q$, ordered by inclusion. When $q=\infty$ this poset becomes the poset of all subgroups of $G$, which we will denote by $\mathcal{L}(G)$.

Consider the functor $F_{q}: \mathcal{N}_{q}(G) \rightarrow G-$ Sets given by $H \mapsto G / H$. In [2] it was proven that if $\Gamma^{q}(G) \neq 1$, then $B(q, G) \simeq \operatorname{hocolim}\left(F_{q}\right)_{h G}$, that is,

$$
B(q, G) \simeq \operatorname{hocolim}_{H \in \mathcal{N}_{q}(G)} B H .
$$

Likewise we have

$$
N B(G) \simeq \operatorname{hocolim}_{H \in \mathcal{L}(G)-\{G\}} B H
$$

The isomorphism $\left(\operatorname{hocolim} F_{q}\right)_{h G} \cong \operatorname{hocolim}\left(F_{q}\right)_{h G}$ (see [6]) shows that

$$
E(q, G) \simeq \underset{H \in \mathcal{N}_{q}(G)}{\operatorname{hocolim}} G / H,
$$

and

$$
E N(G) \simeq \operatorname{hocolim}_{H \in \mathcal{L}(G)-\{G\}} G / H .
$$

Suppose that $H$ and $K$ are subgroups of $G$, so one can see that $x H \subseteq y K$ if and only if, $H \subseteq K$ and $x K=y K$. This allows us to identify the images of the arrows under the functor $F_{q}$ with those of the poset of all proper cosets of $G$. This latter poset is denoted by $\mathcal{C}(G)$.

Definition 2.2. Let $q \geq 2$. We define $\mathcal{C}_{q}(G)$ as the poset of proper cosets of subgroups of $G$ of nilpotency class less than $q$. That is, $\mathfrak{C}_{q}(G)=\left\{x H \in \mathfrak{C}(G) \mid \Gamma^{q}(H)=1\right\}$. When $q=\infty$ this poset becomes the poset of all proper cosets of $G$, that is $\mathcal{C}_{\infty}(G)=\mathfrak{C}(G)$.

Thus, for finite $q$, we have the equivalences: $E(q, G) \simeq \mathcal{C}_{q}(G)$ if $\Gamma^{q}(G) \neq 1$, and $E N(G) \simeq \mathcal{C}(G)$. If $\Gamma^{q}(G)=1$, then $E(q, G) \simeq E G$. But notice that $E G=E(\infty, G)$ is not, in general, homotopy equivalent to $\mathrm{C}(G)$. Note that $B(q, G)$ is always connected since $B_{0}(q, G)=\{1\}$. This implies that $E(q, G)$ is always connected. On the other hand, it was proven in [4] that $\operatorname{EN}(G)$ is connected if and only if $G$ is not isomorphic to cyclic group of order a prime power. So, $G$ is not isomorphic to a cyclic group of prime power order if and only if, $B N(G)$ is connected.

\section{The COSET POSET}

We can make our identification of $E(q, G)$ more efficient by considering only the maximal subgroups of $G$ of nilpotency class $<q$.

Definition 3.1. Let $\mathcal{M}_{q}(G)$ be the poset determined by the maximal nilpotent subgroups of $G$ of class $<q$; that is, any subgroup in $\mathcal{M}_{q}(G)$ is an intersection of maximal nilpotent subgroups of $G$ of class $<q$. The corresponding coset poset is defined as $\mathcal{M C}_{q}(G)=\left\{x H \in \mathcal{C}(G) \mid H \in \mathcal{M}_{q}(G)\right\}$

Recall the following Lemma.

Lemma 3.2 (Quillen, Webb-Thevenaz). Let $f: P \rightarrow Q$ be a $G$-map of $G$-posets. If the subposet $f_{\geq y}=\{x \in P \mid f(x) \geq y\}$ is $G_{y}$-contractible for all $y \in Q$, then $f$ is a homotopy equivalence. 
Remark. The group $G$ acts on the coset poset in two ways: (1) by translation, and (2) by conjugation $g(x H) g^{-1}=g x g^{-1}\left(g H g^{-1}\right)$. We also have the action of $H o l(G)=G \times_{\tau} \operatorname{Aut}(G)$ given by $(g \sigma) \cdot x H=$ $g \sigma(x) \sigma(H)$.

Note that if $f: \mathcal{M C}_{q}(G) \rightarrow \mathfrak{C}_{q}(G)$ denotes the inclusion map, then for $x H \in \mathfrak{C}_{q}(G)$ the poset $f_{\geq x H}$ has an initial element, namely the coset $x M_{H}$, where $M_{H}$ is the intersection of all the subgroups in $\mathcal{M}_{q}(G)$ containing $H$. Moreover, one can see that $x M_{H}$ is fixed by $G_{x H}$ (under both the translation and conjugation action).

We also have (G-equivariant) inclusions $\mathcal{C}_{2}(G) \subseteq \mathcal{C}_{3}(G) \subseteq \cdots \subseteq \mathcal{C}_{q}(G) \subseteq \cdots \subseteq \mathcal{C}(G)$.

Proposition 3.3. For any finite group $G$ such that $\Gamma^{q}(G) \neq 1$, there are homotopy equivalences

$$
E(q, G) \simeq \mathcal{M C}_{q}(G)
$$

and

$$
B(q, G) \simeq \operatorname{hocolim}_{A \in \mathcal{M}_{q}(G)} G / A
$$

We will now focus on computing the Euler characteristic of $E(q, G)$. For this purpose we will use Möbius functions.

Definition 3.4. If $X$ is a finite poset, then the Möbius funtion of this poset is a function $\mu x: X \times X \rightarrow \mathbb{Z}$ determined recursively by the following equations

$$
\mu_{x}(a, a)=1,
$$

and for $a<b$

$$
\mu_{X}(a, b)=-\sum_{a \leq u<b} \mu_{X}(a, u) .
$$

Let $X$ be a subposet of the poset of all subgroups of $G$, and assume that $G$ is in $X$. Then Hall showed that $\mu_{X}(H, G)$ is computed by a signed sum of the number of chains of subgroups in $X$ from $H$ to $G$. We say that $H=K_{0}<K_{1}<\cdots<K_{n}=G$ has length $n$ and it is counted with the sign $(-1)^{n}$. Now, (following Bouc) note that a chain of the form $H=K_{0}<K_{1}<\cdots<K_{n}=G$ corresponds to $|G: H|$ chains of cosets $C_{0}<C_{1}<\cdots<C_{n-1}<G$, where $C_{0}=x H$ and $x$ is a representative of the $|G: H|$ cosets of $H$ in $G$. As the chains $C_{0}<C_{1}<\cdots<C_{n-1}$ form the simplices of the simplicial complex afforded by $\mathcal{C}_{x}$, we see that

$$
1-\chi\left(\bigodot_{x}\right)=\sum_{H \leq G} \mu_{x}(H, G)|G: H|
$$

and thus

$$
\chi\left(\mathcal{C}_{X}\right)=-\sum_{H \in X-\{G\}} \mu_{X}(H, G)|G: H| .
$$

Definition 3.5. Let $q \geq 2$. We define $\mathcal{L}_{q}(G)$ as the poset $\mathcal{N}_{q}(G) \cup\{G\}$, and $\mu_{q}$ as the Möbius function of the poset $\mathcal{L}_{q}(G)$.

Remark. From now on we will assume that $\Gamma^{q}(G) \neq 1$ (in particular $q$ has to be finite). 
Proposition 3.6. Let $G$ be a finite group. Then

$$
\chi(E(q, G))=1-\sum_{H \in \mathcal{L}_{q}(G)} \mu_{q}(H, G)|G: H|=-\sum_{H \in \mathcal{M}_{q}(G)} \mu_{q}(H, G)|G: H| .
$$

Remark. In the formula above the sum can run either over $\mathcal{M}_{q}(G)$ or $\mathcal{N}_{q}(G)$. There is a topological reason for this as we have seen, but there is a combinatorial one which follows from the fact that if $X$ is a suposet of the poset of all subgroups of $G$ (assume $G \in X$ ), then $\mu_{X}(H, G)=0$ if $H$ is not an intersection of maximal subgroups in $X$ (see Theorem 2.3 in [8]). Another important property that facilitates the computation of the Möbius function is that if $\mathcal{X}$ is invariant under $A u t(G)$, then so is $\mu x$.

Example 3.7. A finite group $G$ is said to be TC-group if $[g, h]=1=[h, k]$ implies $[g, k]=1$ for all non-central elements. The poset of maximal abelian subgroups of a TC-group $G$ consists of the maximal abelian subgroups and the center of $G$ which is the intersection of any pair of distinct maximal abelian subgroups of $G$. Then we obtain the formula

$$
1-\chi(E(2, G))=1-|G: Z(G)|+\sum_{1 \leq i \leq N}\left(|G: Z(G)|-\left|G: M_{i}\right|\right)
$$

where $M_{1}, \ldots, M_{N}$ are the maximal abelian subgroups of $G$ (see [2]). The family of TC-groups includes groups such as any non-abelian group of order less than 24, dihedral groups, quaternion groups, and $S L\left(2, \mathbb{F}_{2^{n}}\right)$ with $n \geq 2$. See [11, p. 519 , for a classification of these groups. When $G$ is a TC-group it turns out that $E(2, G)$ has the homotopy type of a wedge of $1-\chi(E(2, G))$ circles (see [2]).

\section{Probability}

P. Hall defined $\phi(G, s)$ as the number of ordered $s$-tuples $\left(g_{1}, \ldots, g_{s}\right) \in G^{s}$ such that $\left\langle g_{1}, \ldots, g_{s}\right\rangle=G$. Thus the probability that a randomly chosen $s$-tuple generates $G$ is given by

$$
P(G, s)=\frac{\phi(G, s)}{|G|^{s}}
$$

Analogously we can define $P_{q}(G, s)$ as the probability that a randomly chosen ordered $s$-tuple generates a nilpotent subgroup of class less than $q$. Of course we have

$$
P_{q}(G, s)=\frac{\left|\operatorname{Hom}\left(F_{s} / \Gamma^{q}, G\right)\right|}{|G|^{s}}
$$

since $\operatorname{Hom}\left(F_{s} / \Gamma^{q}, G\right)=\left\{\left(g_{1}, \ldots, g_{s}\right) \in G^{s} \mid \Gamma^{q}\left(\left\langle g_{1}, \ldots, g_{s}\right\rangle\right)=1\right\}$.

Lemma 4.1. Let $\mu$ be the Möbius function of a poset $\mathcal{X}$, and define the function $\tilde{\mu}$ inductively by

$$
\begin{gathered}
\tilde{\mu}(a, a)=1 \\
\tilde{\mu}(a, b)=-\sum_{a<u \leq b} \mu(u, b) .
\end{gathered}
$$

Then $\mu=\tilde{\mu}$.

Proposition 4.2. Let $\mu_{q}$ be the Möbius function of the poset $\mathcal{L}_{q}(G)=\mathcal{N}_{q}(G) \cup\{G\}$. Then

$$
P_{q}(G, s)=-\sum_{A \in \mathcal{N}_{q}(G)} \frac{\mu_{q}(A, G)}{|G: A|^{s}}
$$


Proof. Note that

$$
\sum_{A \in \mathcal{N}_{q}(G)} \mu_{q}(A, G)|A|^{s}=\sum_{A \in \mathcal{N}_{q}(G)} \sum_{g_{1}, \ldots, g_{s} \in A} \mu_{q}(A, G) .
$$

If we interchange the sums we get

$$
\sum_{A \in \mathcal{N}_{q}(G)} \mu_{q}(A, G)|A|^{s}=\sum_{\left(g_{1}, \ldots, g_{s}\right) \in \operatorname{Hom}\left(F_{s} / \Gamma_{s}^{q}, G\right)} \sum_{\left\langle g_{1}, \ldots, g_{s}\right\rangle \subseteq A} \mu_{q}(A, G),
$$

where the inner sum runs over all $A \in \mathcal{N}_{q}(G)$ containing $\left\langle g_{1}, \ldots, g_{s}\right\rangle$. Recall that $\mu_{q}(G, G)=1$, thus

$$
\sum_{A \in \mathfrak{N}_{q}(G)} \mu_{q}(A, G)|A|^{s}=\sum_{\left(g_{1}, \ldots, g_{s}\right) \in \operatorname{Hom}\left(F_{s} / \Gamma_{s}^{q}, G\right)}\left(-1+\sum_{\left\langle g_{1}, \ldots, g_{s}\right\rangle \subseteq A \subseteq G} \mu_{q}(A, G)\right) .
$$

By the previous Lemma the inner sum is zero. Therefore

$$
\sum_{A \in \mathcal{N}_{q}(G)} \mu_{q}(A, G)|A|^{s}=\sum_{\left(g_{1}, \ldots, g_{s}\right) \in \operatorname{Hom}\left(F_{s} / \Gamma_{s}^{q}, G\right)}(-1)=-\left|\operatorname{Hom}\left(F_{s} / \Gamma_{s}^{q}, G\right)\right| .
$$

The result follows.

Remark. The previous result allows to regard $P_{q}(G, s)$ as a function defined over the complex numbers. The reciprocal function $P_{q}(G, s)$ may be called the probabilistic zeta function of $G$ of class $q$.

Example 4.3. If $G$ is a TC-group with maximal abelian subgroups $M_{1}, \ldots, M_{N}$, then we have

$$
P_{2}(G, s)=\frac{1-N}{|G: Z(G)|^{s}}+\sum_{i=1}^{N} \frac{1}{\left|G: M_{i}\right|^{s}} .
$$

We have the following computations:

(1)

$$
P_{2}\left(\Sigma_{3}, s\right)=\frac{1}{2^{s}}+\frac{3}{3^{s}}-\frac{3}{6^{s}}
$$

(2) If $D_{2 n}$ is the dihedral group of order $2 n$ and $n=2 k \geq 4$, then

$$
P_{2}\left(D_{2 n}, s\right)=\frac{1}{2^{s}}+\frac{k}{k^{s}}-\frac{k}{n^{s}} \text {. }
$$

Whereas from [5] we have

$$
P_{2}\left(A_{4}, s\right)=\frac{1}{3^{s}}+\frac{4}{4^{s}}-\frac{4}{12^{s}} .
$$

$$
\begin{gathered}
P\left(A_{5}, s\right)=1-\frac{5}{5^{s}}-\frac{6}{6^{s}}-\frac{10}{10^{s}}+\frac{20}{20^{s}}+\frac{60}{30^{s}}-\frac{60}{60^{s}} . \\
P_{2}\left(\Sigma_{4}, s\right)=\frac{7}{6^{s}}+\frac{4}{8^{s}}-\frac{6}{12^{s}}-\frac{4}{24^{s}} . \\
P_{2}(P S L(2,7), s)=\frac{8}{24^{s}}+\frac{35}{42^{s}}+\frac{28}{56^{s}}-\frac{42}{84^{s}}-\frac{28}{168^{s}} .
\end{gathered}
$$


$(7)$

$$
P_{3}\left(\Sigma_{4}, s\right)=\frac{3}{3^{s}}-\frac{2}{6^{s}}+\frac{4}{8^{s}}-\frac{4}{24^{s}} .
$$

Proposition 4.4. If $q \geq 2$, then $\chi(E(q, G))=P_{q}(G,-1)$.

Corollary 4.5. Let $m_{q}(G)$ be the greatest common divisor of the indices of the maximal subgroups in $\mathcal{N}_{q}(G)$. Then $m_{q}(G)$ divides $\chi(E(q, G))$.

Example 4.6. If $G$ is a non-abelian $p$-group, then $\chi(E(2, G))$ is divisible by $p^{\alpha}$ where $p^{\alpha}$ is the index of a maximal abelian subgroup of $G$.

Lets consider the simple group $P S L(2, p)$ where $p$ is a prime number greater than 3 . The order of $\operatorname{PSL}(2, p)$ is $2 p q r$ where $q=\frac{1}{2}(p-1)$ and $r=\frac{1}{2}(p+1)$. The numbers $p, q, r$ are relatively prime. The maximal abelian subgroups of $P S L(2, p)$ are $\mathbb{Z} / p, \mathbb{Z} / q, \mathbb{Z} / r$ and $D_{4}$, where $D_{2 n}$ is the dihedral group of order $2 n$.

Proposition 4.7. Let $p$ be a prime number greater than 3. Then

$$
P_{2}(P S L(2, p), s)=\frac{p+1}{(2 q r)^{s}}+\frac{p r}{(2 p r)^{s}}+\frac{p q}{(2 p q)^{s}}+\frac{\frac{1}{6} p q r}{\left(\frac{1}{2} p q r\right)^{s}}-\frac{\frac{1}{2} p q r}{(p q r)^{s}}-\frac{p+p r+p q-\frac{p q r}{3}}{(2 p q r)^{s}} .
$$

Remark. Notice that the isomorphism $\operatorname{Hom}(A, B \times C) \cong \operatorname{Hom}(A, B) \times \operatorname{Hom}(A, C)$ induces a homeomorphism $E(q, G \times H) \cong E(q, G) \times E(q, H)$, and produces the identity

$$
P_{q}(G \times H, s)=P_{q}(G, s) P_{q}(H, s) .
$$

This tells us that

$$
\chi(E(q, G \times H))=\chi(E(q, G)) \chi(E(q, H)),
$$

which is is simpler than the formula obtained in [4] for the poset of proper cosets. What makes the ordinary coset poset more complex with respect to products is that we do not have, in general, an isomorphism between $B N_{n}(G \times H)$ and $B N_{n}(G) \times B N_{n}(H)$.

This latter formula allows to compute the Euler characteristic of $\mathcal{C}_{q}(G)$ for any nilpotent group $G$ in terms of that of its Sylow supgroups.

Example 4.8. Let $G$ be the central product of $Q_{8}$ with itself. $G$ is an extraspecial group of order 32 . The maximal abelian subgroups of $G$ have order 8 and the center of $G$ is cyclic of order 2. It follows that $E(2, G)$ has the homotopy type of a 2-dimensional complex. We can see that

$$
\mu_{2}(H, G)=\left\{\begin{array}{rll}
-1 & \text { if } & |H|=8 \\
2 & \text { if } & |H|=4 \\
-16 & \text { if } & |H|=2
\end{array}\right.
$$

and find that $\chi(E(2, G))=76$. We also have

$$
P_{2}(G, s)=\frac{15}{4^{s}}-\frac{30}{8^{s}}+\frac{16}{16^{s}}
$$

We can also compute the homology of $E(2, G)$ by using MAGMA: 


$$
H_{*}(E(2, G) ; \mathbb{Z})=\left\{\begin{array}{cll}
\mathbb{Z} & \text { if } & *=0 \\
\mathbb{Z} / 2 & \text { if } & *=1 \\
\mathbb{Z}^{75} & \text { if } & *=2 \\
0 & \text { if } & * \geq 3 .
\end{array}\right.
$$

Note that this is the same as the homology of $\mathbb{R} P^{2} \vee \bigvee^{75} S^{2}$.

Remark. This latter example shows that $E(q, G)$ does not have to have the homotopy type of a bouquet of spheres when $G$ is solvable. In fact, if it was a bouquet of spheres of dimension $>1$, then $\pi_{1}(B(2, G))=G$.

Remark. Note that $P_{q}(G, 1)=1$ for all $G$. Define

$$
R_{q}(G, S)=1-P_{q}(G, s)=\sum_{\mathcal{N}_{q}(G) \cup\{G\}} \frac{\mu_{q}(H, G)}{|G: H|^{s}}
$$

Thus $\left.-R_{q}(G,-1)=\tilde{\chi}\left(\mathcal{C}_{q} G\right)\right)$, the reduced Euler characteristic of $\mathcal{C}_{q}(G)$. This function $R_{q}(G, S)$ has a probabilistic interpretation: $R_{q}(G, s)$ is the probability that a randomly chosen ordered $s$-tuple does not generate a nilpotent subgroup of class $<q$. The inclusions $\operatorname{Hom}\left(F_{n} / \Gamma_{n}^{q}, G\right) \subseteq \operatorname{Hom}\left(F_{n} / \Gamma_{n}^{q+1}, G\right)$ show that for all integers $s \geq 1$ we have

$$
R_{2}(G, s) \geq \cdots \geq R_{q}(G, s) \geq R_{q+1}(G, s) \geq \cdots
$$

Likewise, the function $P_{q}(G, s)-P_{q-1}(G, s)$ is the probability that a randomly chosen $s$-tuple generates a nilpotent subgroup of class $q-1$.

Remark. If $G$ is finite, then the series $P_{q}(G, s)$ stabilize, that is, there is some $q_{G}$ such that $P_{q_{G}}(G, s)=$ $P_{q}(G, s)$ for all $q \geq q_{G}$. The integer $q_{G}$ is the smallest integer so that $\Gamma^{q_{G}}(H)=1$ for all proper, nilpotent subgroups of $G$.

4.1. Factorizations. The ring of finite Dirichlet series with integer coefficients

$$
\mathcal{R}=\left\{\sum_{n \geq 0} \frac{a_{n}}{n^{s}}: a_{n} \in \mathbb{Z}, a_{n} \neq 0 \text { for finitely many } n \text { 's }\right\}
$$

is a unique factorization domain as it is a polynomial ring over $\mathbb{Z}$ in $\frac{1}{2^{s}}, \frac{1}{3^{s}}, \frac{1}{5^{s}}, \ldots$ It is then natural to study the factorization of $P_{q}(G, s)$ and $R_{q}(G, s)$ in $\mathcal{R}$.

Example 4.9. (1) Recall that $D_{2 n}$ is nilpotent if and only if $n$ is a power of 2. Suppose that $n=2^{r}$ and $r>1$, so $D_{2 n}$ has class $r$. Then

$$
R_{r-k+1}\left(D_{2 n}, s\right)=1-\frac{1}{2^{s}}-\frac{2^{k}}{2^{k s}}+\frac{2^{k}}{\left(2^{k+1}\right)^{s}}=-\left(1-\frac{1}{2^{s}}\right) \prod_{d \mid k} \Phi_{d}\left(\frac{2}{2^{s}}\right),
$$

where $\Phi_{d}$ is the $d^{t h}$ cyclotomic polynomial, and $1 \leq r-k<r$. Moreover,

$$
E\left(r-k+1, D_{2 n}\right) \simeq \bigvee_{2^{2 k}-1} S^{1}
$$

for $1 \leq r-k<r$. 
(2) If $G$ is the central product of $Q_{8}$ with itself, then

$$
R_{2}(G, s)=\left(1-\frac{1}{2^{s}}\right)\left(1-\frac{2}{2^{s}}\right)\left(1+\frac{3}{2^{s}}-\frac{8}{4^{s}}\right) .
$$

(3) If $q=2^{n}$ and $n \geq 2$, then

$$
R_{2}(S L(2, q), s)=1+\frac{q(q+1)}{[q(q-1)]^{s}}-\frac{q+1}{\left[q^{2}-1\right]^{s}}-\frac{\frac{1}{2} q(q-1)}{[q(q-1)]^{s}}-\frac{\frac{1}{2} q(q+1)}{[q(q+1)]^{s}}
$$

is irreducible.

(4) If $M_{11}$ is the Mathieu group of degree 11, then

$$
R_{2}\left(M_{11}, s\right)=1-\frac{144}{720^{s}}-\frac{55}{880^{s}}-\frac{495}{990^{s}}-\frac{660}{1320^{s}}-\frac{396}{1584^{s}}+\frac{330}{1980^{s}}+\frac{660}{2640^{s}}+\frac{1980}{3960^{s}}-\frac{561}{7920^{s}}
$$

is irreducible.

Conjecture 4.10. If $G$ is a finite simple group, then $R_{2}(G, s)$ is irreducible in $\mathcal{R}$.

Note that if $G$ is nilpotent then $P_{q}(G, s)$ is reducible for all finite $q \geq 2$. More precisely, if the Sylow subgroups of $G$ are $P_{1}, \ldots, P_{n}$, then $P_{q}(G, s)=P_{q}\left(P_{1}, s\right) \cdots P_{q}\left(P_{n}, s\right)$.

4.2. Lifting nilpotent tuples. Let $H \triangleleft G$. Recall that the ordinary probabilistic zeta function satisfies $P(G, s)=P(G / N, s) P(G, N, s)$, where $P(G, N, s)$ is the probability that a random lift of a generating $s$-tuple is $G / N$ to an $s$-tuple in $G$ generates $G$ (see [4]). Note that this is a conditonal probability formula. We will say that a $s$-tuple is $q$-nilpotent if the subgroup that it generates is nilpotent of class $<q$. We will compute the probability that a random lift of a $q$-nilpotent $s$-tuple in $G / N$ is a $q$-nilpotent $s$-tuple in $G$. This latter will be denoted by $P_{q}(G, N, s)$.

Let $\phi \in \operatorname{Hom}\left(F_{n} / \Gamma_{n}^{q}, G / N\right)$ and $K \subseteq G$. Define $\alpha_{q}(K, \phi)$ as the number of lifts of $\phi$ to $q$-tuples in $K$, and $\beta_{q}(K, \phi)$ as the number of such lifts that generate $K$. Thus

$$
\alpha_{q}(K, \phi)=\sum_{H \in \mathcal{L}_{q}(K)} \beta_{q}(H, \phi)
$$

and so

$$
\beta_{q}(K, \phi)=\sum_{H \in \mathcal{L}_{q}(K)} \mu_{q}(H, K) \alpha_{q}(H, \phi) .
$$

Note that $\beta_{q}(G)=0$ (if $G$ is not nilpotent of class less that $q$ ). Moreover, $\alpha_{q}(H, \phi)$ is equal to $|H \cap N|^{s}$ if $\phi$ is in $H N / N$, or zero otherwise. So

$$
\alpha_{q}(G, \phi)=-\sum_{\substack{H \in \mathcal{N}_{q}(K) \\ \phi \in H N / N}} \mu_{q}(H, G)|H \cap N|^{s}
$$

Note that $\frac{\alpha_{q}(G, \phi)}{|N|^{s}}$ is the probability that a randomly chosen lift of $\phi$ is a $q$-tuple in $G$. This latter depends on $\phi$, in general, unless $q=\infty$. 
Therefore, the total number of lifts of $q$-tuples in $G / N$ to $q$-tuples in $G$ is given by

$$
\begin{aligned}
\Phi_{q}(G, N, s) & =\sum_{\phi \in H o m\left(F_{n} / \Gamma_{n}^{q}, G / N\right)} \alpha_{q}(G, \phi) \\
& =-\sum_{\phi \in H o m\left(F_{n} / \Gamma_{n}^{q}, G / N\right)} \sum_{H \in \mathcal{N}_{q}(K)} \mu_{q}(H, G)|H \cap N|^{s} \\
& =-\sum_{H \in \mathcal{N}_{q}(K)} \mu_{\phi \in H o m\left(F_{n} / \Gamma_{n}^{q}, H N / N\right)} \mu_{q}(H, G)|H \cap N|^{s} \\
& =-\sum_{H \in \mathcal{N}_{q}(K)} \mu_{q}(H, G)|H|^{s} .
\end{aligned}
$$

Thus

$$
P_{q}(G, N, s)=\frac{\Phi_{q}(G, N, s)}{|N|^{s}\left|\operatorname{Hom}\left(F_{n} / \Gamma_{n}^{q}, G / N\right)\right|}=\frac{\Phi_{q}(G, N, s) /|G|^{s}}{P_{q}(G / N, s)}=\frac{P_{q}(G, s)}{P_{q}(G / N, s)} .
$$

Unlike the case of the ordinary probabilistic function of $G$, it is not necessarily true that $P(G, N, s)$ will be a Dirichlet series in $\mathcal{R}$. This latter can be seen by looking at the values of $P_{q}(G, s)$ and $P_{q}(G / N, s)$ at $s=-1$ (see, for instance, Example 4.3). Thus $P_{q}(G, N, s)$ is rational.

4.3. Localizations. We can also consider localizations of the function $P_{q}(G, s)$ at different primes. There are at least three "local" versions of the probabilistic functions.

- Let $p$ be a prime number and let $\mathcal{N}_{q}(G)_{p}=\left\{\left.H \in \mathcal{N}_{q}(G)|| G\right|_{p}=|H|_{p}\right\}$. Define

$$
P_{q}(G, s)_{p}=-\sum_{H \in \mathcal{N}_{q}(G)_{p}} \frac{\mu_{q}(H, G)}{|G: H|^{s}} .
$$

This latter series is the image of $P_{q}(G, s)$ in the localization of $\mathcal{R}$ at $\frac{1}{p^{s}}$. Thus $R_{q}(G, s)_{p}=$ $1-P_{q}(G, s)_{p}$.

- We can twist again Hall's question and this time ask the following: given a fixed prime $p$, what is the probability that a randomly chosen $s$-tuple of $G$ generates an abelian $p$-subgroup? This is

$$
\frac{\operatorname{Hom}\left(\mathbb{Z}_{p}, G\right)}{|G|^{s}}
$$

where $\mathbb{Z}_{p}$ stands for the $p$-adic integers. This probability can be described by using the poset of abelian $p$-subgroups of $G$. Note that if the maximal abelian $p$-subgroups of $G$ are maximal abelian then this probability function coincides with $P_{2}(G, s)_{p}$ (for example, take $G=S L\left(2, \mathbb{F}_{2^{n}}\right)$ ).

- Let $\mathcal{N}_{q}(G)_{\{p\}}=\left\{H \in \mathcal{N}_{q}(G):|G: H|=p^{k}\right.$, for some $\left.k \geq 1\right\}$. We define

$$
P_{q}(G, s)_{\{p\}}=-\sum_{H \in \mathcal{N}_{q}(G)_{\{p\}}} \frac{\mu_{q}(H, G)}{|G: H|^{s}} .
$$

Thus

$$
P_{q}(G, s)_{\{p\}}=-\sum_{k \geq 1} \frac{a_{k}}{p^{k s}},
$$

where

$$
a_{k}=\sum_{\substack{H \in \mathcal{N}_{q}(G) \\|G: H|=p^{k}}} \mu_{q}(H, G) .
$$




\section{Commuting tuples in the Symmetric Group}

Note that $\left|\operatorname{Hom}\left(\mathbb{Z}^{2}, \Sigma_{n}\right)\right|=n ! p(n)$, where $p(n)$ is the number of partitions of $n$. Thus

$$
P_{2}\left(\Sigma_{n}, 2\right)=\frac{p(n)}{n !} .
$$

Computing the number of commuting $s$-tuples in $\Sigma_{n}$ is more elaborate than the previous observation as we will see. Let $\mathcal{P}_{n}$ be the set of all partitions of $n$. A partition of $n$ will be denoted by $1^{a_{1}} 2^{a_{2}} \cdots n^{a_{n}}$, where $a_{k}$ is the number of $k$-cycles in the partition. To compute $\left|H o m\left(\mathbb{Z}^{s}, \Sigma_{n}\right)\right|$ one has to note that an element in $\operatorname{Hom}\left(\mathbb{Z}^{s}, \Sigma_{n}\right)$ can be identified with a $\mathbb{Z}^{s}$-set of degree $n$. A $\mathbb{Z}^{s}$-set of degree $n$ decomposes into transitive $\mathbb{Z}^{s}$-sets of degree $a_{i}$ so that $1^{a_{1}} 2^{a_{2}} \cdots n^{a_{n}}$ is a partition of $n$. A transitive $\mathbb{Z}^{s}$-set of degree $r$ can be identified with a subgroup of $\mathbb{Z}^{s}$ of index $r$. Let $j_{r}\left(\mathbb{Z}^{s}\right)$ be the number of subgroups of $\mathbb{Z}^{s}$ of index $r$. The number $j_{r}\left(\mathbb{Z}^{s}\right)$ turns out to be finite for all $s$ and $r$, and can be computed by using the following two formulae (see [12, and [10, Section 15. 2):

$$
j_{r}\left(\mathbb{Z}^{s}\right)=\sum_{r_{1} \cdots r_{s}=r} r_{2} r_{3}^{2} \cdots r_{s}^{s-1},
$$

and

$$
j_{r}\left(\mathbb{Z}^{s}\right)=\sum_{d \mid r} j_{d}\left(\mathbb{Z}^{s-1}\right)
$$

Thus we have the following result.

Proposition 5.1. The number of commuting s-tuples in $\Sigma_{n}$ is given by

$$
\left|H o m\left(\mathbb{Z}^{s}, \Sigma_{n}\right)\right|=\sum_{1^{a_{1}} 2^{a_{2} \ldots n^{a_{n}} \in \mathcal{P}_{n}}} \frac{n !}{\prod_{i=1}^{n} i^{a_{i}} a_{i} !} \prod_{i=1}^{n} j_{i}\left(\mathbb{Z}^{s}\right)^{a_{i}} .
$$

6. Conjugacy Classes, LOWER Bounds, AND Upper Bounds.

Another important invariant of a group is the number of conjugacy classes of ordered commuting $n$-tuples. If $n \geq 1$, then we denote by $k_{n}(G)$ the number of conjugacy classes of commuting $n$-tuples in $G$. In this section we will use the letter $n$ instead of $s$ to stress the fact that all the results concerning $P_{2}$ are for non-negative integers. We begin with the following useful lemma.

Lemma 6.1. Let $K$ be any discrete group. Then

$$
|\operatorname{Hom}(K \times \mathbb{Z}, G)| /|G|=|\operatorname{Hom}(K, G) / G| .
$$

Proof. Note that

$$
\operatorname{Hom}(K \times \mathbb{Z}, G)=\bigsqcup_{\phi \in H o m(K, G)} C_{G}(\phi) .
$$

So

$$
\begin{aligned}
\frac{1}{|G|}|\operatorname{Hom}(K \times \mathbb{Z}, G)| & =\frac{1}{|G|} \sum_{\phi \in H o m(K, G)}\left|C_{G}(\phi)\right|=\sum_{\phi \in \operatorname{Hom}(K, G)} \frac{1}{\left|G: C_{G}(\phi)\right|} \\
& =\sum_{(\phi) \in \operatorname{Hom}(K, G) / G} 1=|\operatorname{Hom}(K, G) / G| .
\end{aligned}
$$


The previous result shows that

$$
k_{n}(G)=\left|\operatorname{Hom}\left(\mathbb{Z}^{n}, G\right) / G\right|=\left|\operatorname{Hom}\left(\mathbb{Z}^{n+1}, G\right)\right| /|G|=P_{2}(G, n+1)|G|^{n} .
$$

This latter in turn shows that

$$
k_{n}(G)=-\frac{1}{|G|} \sum_{H \in \mathcal{N}_{2}(G)} \mu_{2}(H, G)|H|^{n+1} .
$$

Proposition 6.2. Let $H$ be a subgroup of $G$. Then

$$
|G: H|^{-2 n} P_{2}(H, n) \leq P_{2}(G, n) \leq P_{2}(H, n),
$$

and therefore

$$
|G: H|^{-1} k_{n}(H) \leq k_{n}(G) \leq|G: H|^{n} k_{n}(H) .
$$

Proof. We proceed by induction on $n$ to prove all the inequalities. The case $n=1$ is well-known (see [7]). Suppose that $n>1$. For the right-hand side inequalities we have

$$
\left|\operatorname{Hom}\left(\mathbb{Z}^{n}, G\right)\right|=\sum_{g \in G}\left|\operatorname{Hom}\left(\mathbb{Z}^{n-1}, C_{G}(g)\right)\right| \leq \sum_{g \in G}\left|C_{G}(g): C_{H}(g)\right|^{n-1}\left|\operatorname{Hom}\left(\mathbb{Z}^{n-1}, C_{H}(g)\right)\right|
$$

Since $\left|C_{G}(g): C_{H}(g)\right| \leq|G: H|$ it follows that

$$
\left|\operatorname{Hom}\left(\mathbb{Z}^{n}, G\right)\right| \leq \sum_{g \in G}|G: H|^{n-1}\left|\operatorname{Hom}\left(\mathbb{Z}^{n-1}, C_{H}(g)\right)\right|
$$

On the other hand,

$$
\begin{aligned}
\sum_{g \in G}\left|\operatorname{Hom}\left(\mathbb{Z}^{n-1}, C_{H}(g)\right)\right| & =\sum_{\phi \in H o m\left(\mathbb{Z}^{n-1}, H\right)}\left|C_{G}(\phi)\right| \\
& =\sum_{[\phi] \in \operatorname{Hom}\left(\mathbb{Z}^{n-1}, H\right) / H}\left|C_{G}(\phi)\right|\left|H: C_{H}(\phi)\right| \\
& =\sum_{[\phi] \in \operatorname{Hom}\left(\mathbb{Z}^{n-1}, H\right) / H}|H|\left|C_{G}(\phi): C_{H}(\phi)\right| \\
& \leq \sum_{[\phi] \in \operatorname{Hom}\left(\mathbb{Z}^{n-1}, H\right) / H}|H||G: H| \\
& =|G| k_{n-1}(H) \\
& =|G: H|\left|H o m\left(\mathbb{Z}^{n}, H\right)\right| .
\end{aligned}
$$

The right-hand side inequalities follow.

For the left-hand side inequalities we have

$$
\begin{aligned}
|H|^{n} \mid \operatorname{Hom}\left(\mathbb{Z}^{n}, H\right) & =\sum_{h \in H}\left|\operatorname{Hom}\left(\mathbb{Z}^{n-1}, H\right)\right| \leq|H|^{n} \sum_{h \in H}\left|C_{G}(h): C_{H}(h)\right|^{n-1} \mid \operatorname{Hom}\left(\mathbb{Z}^{n-1}, C_{G}(h) \mid\right. \\
& \leq|H|^{n}|G: H|^{n-1} \sum_{h \in H} \mid \operatorname{Hom}\left(\mathbb{Z}^{n-1}, C_{G}(h) \mid\right. \\
& \leq|G|^{n-1}|H| \sum_{g \in G} \mid \operatorname{Hom}\left(\mathbb{Z}^{n-1},\left.C_{G}(g)|\leq| G\right|^{n}\left|\operatorname{Hom}\left(\mathbb{Z}^{n}, G\right)\right|\right.
\end{aligned}
$$


The left-hand side inequalities follow.

Remark. Since $P_{2}(G \times H, n)=P_{2}(G, n) P_{2}(H, n)$, it follows that $k_{n}(G \times H)=k_{n}(G) k_{n}(H)$.

Proposition 6.3. Let $N \triangleleft G$. Then $P_{2}(G, n) \leq P_{2}(G / N, n) P_{2}(N, n)$, and therefore

$$
k_{n}(G) \leq k_{n}(G / N) k_{n}(N) .
$$

Proof. We proceed by induction on $n$. The result for $n=1$ is well-known. For $n>1$ we have

$$
\begin{aligned}
\left|\operatorname{Hom}\left(\mathbb{Z}^{n}, G\right)\right| & =\sum_{g \in G}\left|\operatorname{Hom}\left(\mathbb{Z}^{n-1}, C_{G}(g)\right)\right| \\
& \leq \sum_{g \in G}\left|\operatorname{Hom}\left(\mathbb{Z}^{n-1}, C_{G}(g) N / N\right)\right|\left|\operatorname{Hom}\left(\mathbb{Z}^{n-1}, C_{N}(g)\right)\right| \\
& \leq \sum_{g \in G} \mid \operatorname{Hom}\left(\mathbb{Z}^{n-1}, C_{G / N}(g N)|| \operatorname{Hom}\left(\mathbb{Z}^{n-1}, C_{N}(g)\right) \mid\right. \\
& =\sum_{x N \in G / N} \sum_{g \in x N} \mid \operatorname{Hom}\left(\mathbb{Z}^{n-1}, C_{G / N}(g N)|| \operatorname{Hom}\left(\mathbb{Z}^{n-1}, C_{N}(g)\right) \mid\right. \\
& =\sum_{x N \in G / N} \mid \operatorname{Hom}\left(\mathbb{Z}^{n-1}, C_{G / N}(x N)\left|\sum_{g \in x N}\right| \operatorname{Hom}\left(\mathbb{Z}^{n-1}, C_{N}(g)\right) \mid .\right.
\end{aligned}
$$

On the other hand

$$
\sum_{g \in x N}\left|\operatorname{Hom}\left(\mathbb{Z}^{n-1}, C_{N}(g)\right)\right|=\sum_{\phi \in H o m\left(\mathbb{Z}^{n-1}, N\right)}\left|C_{x N}(\phi)\right| .
$$

Note that if $y$ is in $C_{x N}(\phi)$, then $C_{x N}(\phi)=C_{G}(\phi) \cap x N=y C_{G}(\phi) \cap y N=y C_{N}(\phi)$. So $C_{x N}(\phi)$ is either empty or a coset of $C_{N}(\phi)$. Thus

$$
\begin{aligned}
\sum_{g \in x N}\left|\operatorname{Hom}\left(\mathbb{Z}^{n-1}, C_{N}(g)\right)\right| & \leq \sum_{\phi \in \operatorname{Hom}\left(\mathbb{Z}^{n-1}, N\right)}\left|C_{N}(\phi)\right| \\
& =\sum_{[\phi] \in \operatorname{Hom}\left(\mathbb{Z}^{n-1}, N\right) / N}|N| \\
& =|N| k_{n-1}(N)=\left|\operatorname{Hom}\left(\mathbb{Z}^{n}, N\right)\right| .
\end{aligned}
$$

The result follows.

The following result provides a slight improvement over that in Lemma 2 of [7].

Proposition 6.4. Let $x$ be an element of $G-Z(G)$ so that $\left|C_{G}(x)\right|$ is as large as possible, and let $p$ be the smallest prime divisor of the index of $Z(G)$ in $G$. Let $c$ denote $\left|C_{G}(x)\right|$. Then for $n \geq 1$,

$$
P_{2}(G, n+1) \leq \frac{\left(p^{n}+\cdots+p+1\right) c^{n}}{p^{n}|G|^{n}} .
$$


Proof. If $\phi=\left(x_{1}, \ldots, x_{n}\right)$ is in $\operatorname{Hom}\left(\mathbb{Z}^{n}, G\right)$, then $C_{G}(\phi) \subseteq C_{G}\left(x_{i}\right)$. If at least one of the components of $\phi$ is not in $Z(G)$, then $\left|C_{G}(\phi)\right| \leq c$. Then for $n \geq 1$ we have

$$
\left|H o m\left(\mathbb{Z}^{n}, G\right)\right| \geq|Z(G)|^{n}+\left(k_{n}(G)-|Z(G)|^{n}\right) \frac{|G|}{c} .
$$

Note that $c /|Z(G)| \geq p$. So

$$
\begin{aligned}
P_{2}(G, n+1) & \leq \frac{c}{|G|} P_{2}(G, n)+\left(1-\frac{c}{|G|}\right) \frac{|Z(G)|^{n}}{|G|^{n}} \\
& \leq \frac{c}{|G|} P_{2}(G, n)+\frac{|Z(G)|^{n}}{|G|^{n}} \\
& \leq \frac{c}{|G|} P_{2}(G, n)+\frac{c^{n}}{p^{n}|G|^{n}} .
\end{aligned}
$$

An inductive argument applied to the latter inequality completes the proof.

Proposition 6.5. Let $p$ be the smallest prime divisor of $|G: Z(G)|$, and let $m$ be the index of $Z(G)$ in G. Then

$$
\frac{m n+m-n}{m^{n+1}} \leq P_{2}(G, n+1) \leq \frac{p^{n+1}+p^{n}-1}{p^{2 n+1}} .
$$

Proof. If $p$ is the smallest prime divisor of $|G|$, then $\left|G: C_{G}(\phi)\right| \geq p$ for all $\phi \notin Z(G)^{n}$. So

$$
\left|H o m\left(\mathbb{Z}^{n}, G\right)\right| \geq|Z(G)|^{n}+p\left(k_{n}(G)-|Z(G)|^{n}\right),
$$

and thus

$$
k_{n}(G) \leq \frac{1}{p}\left(\left|\operatorname{Hom}\left(\mathbb{Z}^{n}, G\right)\right|+(p-1)|Z(G)|^{n}\right)
$$

Dividing by $|G|^{n}$ we have

$$
P_{2}(G, n+1) \leq \frac{1}{p}\left(P_{2}(G, n)+(p-1) \frac{1}{|G: Z(G)|^{n}}\right) .
$$

Note that $|G: Z(G)| \geq p^{2}$, else $G / Z$ is a cyclic group of order $p$. So

$$
P_{2}(G, n+1) \leq \frac{1}{p}\left(P_{2}(G, n)+\frac{p-1}{p^{2 n}}\right) .
$$

An inductive argument applied to the latter inequality completes the proof for the right hand side inequality. The left hand side inequality is obtained in a similar way.

In the last result $p$ is of course at least 2 . The same proof yields the following result proved in 9 .

Corollary 6.6. If $G$ is a nonabelian group, then

$$
P_{2}(G, n+1) \leq \frac{3 \cdot 2^{n}-1}{2^{2 n+1}} .
$$

We close this section by providing a family of congruences for $k_{n}(G)$. The congruence for $k_{1}(G)$ was proved in [3].

Proposition 6.7. Let $p_{1}, \ldots, p_{l}$ be the prime divisors of $|G|$, and let $D_{n}$ be the greatest common divisor of $p_{1}^{n}-1, \ldots, p_{l}^{n}-1$. Then

$$
k_{n-1}(G) \equiv|G|^{n-1} \quad\left(\bmod D_{n}\right) .
$$


The idea to prove this is as follows: first recall that

$$
\sum_{H \in \mathcal{L}_{2}(G)} \mu_{2}(H, G)=0
$$

So

$$
-|G| k_{n-1}(G)=-|G|^{n}+\sum_{H \in \mathcal{L}_{2}(G)} \mu_{2}(H, G)\left(|H|^{n}+a\right)
$$

for any number $a$. Let us restrict to the case $a=-1$. Thus, the last three equations show that if $d$ divides $|H|^{n}-1$ for all $H \subseteq G$, then $k_{n-1}|G| \equiv|G|^{n}(\bmod d)$. If $D_{n}$ is as defined in the proposition, then one can see that $D_{n}$ is relatively prime to $|G|$ and that it divides $|H|^{n}-1$ for all $H \subseteq G$. This yields the desired congruence.

\section{REFERENCES}

[1] A. Adem, F. Cohen, Commuting Elements and Spaces of Homomorphisms, Mathematische Annalen 338 (2007), $587-626$.

[2] A. Adem, F. Cohen, E. Torres-Giese Commuting elements, simplicial spaces and filtrations of classigying spaces. arXiv:0901.0137.

[3] A. Mann, Conjugacy classes in finite groups. Israel J. of Math. 31 (1978), no. 1, 78-84.

[4] K. S. Brown, The coset poset and probabilistic zeta function of a finite group. J. Algebra 225 (2000), no. 2, 989-1012.

[5] N. A. Boston, A probabilistic generalization of the Riemann zeta function. Analytic number theory, Vol. 1, 155-162, Progr. Math., 138, Birkhuser Boston, Boston, MA, 1996.

[6] W. Dwyer, Homotopy theoretic methods in group cohomology. Advanced Courses in Mathematics. CRM Barcelona. Birkhuser Verlag, Basel, 2001.

[7] R. Guralnick, G. Robinson, On the commuting probability in finite groups. Journal of Algebra 300 (2006), 509-528.

[8] P. Hall, The eulerian functions of a group. Quart. J. Math. 7 (1936), 134-151.

[9] P. Lescot, Isoclinism classes and commutativity degrees of finite groups. Journal of Algebra 177 (1995), 847-869.

[10] A. Lubotzky, D. Segal, Subgroup growth. Progress in Mathematics, 212. Birkhuser Verlag, Basel, 2003.

[11] R. Schmidt, Subgroup lattices of groups, de Gruyter Expositions in Mathematics 14, Berlin, 1994.

[12] L. Solomon, Partially ordered sets with colors. Relations between combinatorics and other parts of mathematics, pp. 309-329, Proc. Sympos. Pure Math., XXXIV, Amer. Math. Soc., Providence, R.I., 1979.

Department of Mathematics, University of Michigan, Ann Arbor Mi 48109, USA

E-mail address: etorresg@umich.edu 